Erratum

\title{
Corrigendum to "Multidimensional endogenous gridpoint method: Solving triangular dynamic stochastic optimization problems without root-finding operations" [Econom. Lett. 135 (2015) 72-76]
}

\section{Fedor Iskhakov}

Research School of Economics, The Australian National University, ACT 2601, Australia

\section{A R T I C L E I N F O}

Assumption A1 in Iskhakov (2015) is incomplete. The correct version should read:

Assumption A1. Transition rules for the continuous state variables $X_{t}^{1}, \ldots, X_{t}^{M}, j \in\{1, \ldots, M\}$ can be expressed in the form

$X_{t+1}^{j}=\chi^{j}\left(f^{1}\left(X_{t}^{1}, x_{t}^{1}, s_{t}\right), f^{2}\left(X_{t}^{1}, x_{t}^{1}, X_{t}^{2}, x_{t}^{2}, s_{t}\right), \ldots\right.$,

$$
\left.f^{M}\left(X_{t}, x_{t}, s_{t}\right), s_{t}, s_{t+1}, \xi_{t+1}\right),
$$

such that the post-decision states $f(t)=\left(f^{1}(t), \ldots, f^{M}(t)\right) \in \mathbb{R}^{M}$ admit the following structure

$f^{1}(t)=f^{1}\left(X_{t}^{1}, x_{t}^{1}, s_{t}\right)$,

$f^{2}(t)=f^{2}\left(X_{t}^{1}, x_{t}^{1}, X_{t}^{2}, x_{t}^{2}, s_{t}\right)$,

$f^{M}(t)=f^{M}\left(X_{t}^{1}, x_{t}^{1}, \ldots, X_{t}^{M}, x_{t}^{M}, s_{t}\right)=f^{M}\left(X_{t}, x_{t}, s_{t}\right)$,

where $\chi^{j}(\cdot)$ and $f^{j}(\cdot)$ are deterministic differentiable functions, the partial derivatives of $f^{j}(t)$ take the form

$$
\begin{aligned}
\frac{\partial f^{j}(t)}{\partial x_{t}^{i}} & =\frac{\partial f^{j}\left(X_{t}^{1}, x_{t}^{1}, \ldots, X_{t}^{j}, x_{t}^{j}, s_{t}\right)}{\partial x_{t}^{i}} \\
& =g_{i}^{j}\left(f^{1}(t), \ldots, f^{j}(t), s_{t}\right), \quad i \leq j
\end{aligned}
$$

for each $j=1 \ldots$ M and arbitrary functions $g_{i}^{j}$, and it holds $\frac{\partial f^{j}(t)}{\partial x_{t}^{j}} \neq 0$ and $\frac{\partial f^{j}(t)}{\partial X_{t}^{j}} \neq 0 ; \xi_{t+1} \in \mathbb{R}^{K}$ are idiosyncratic shocks.

The additional condition ( 3 ) ensures that $\frac{\partial f^{k}(t)}{\partial x_{t}^{j}}$ does not depend on the continuous state and decision variables in period $t$ other than through the post decision states. These partial derivatives had been implicitly treated as constants in Section 3. I apologize for the error and express my gratitude to Jeppe Druedahl and Thomas Jørgensen at the University of Copenhagen for pointing it out.

With the above correction, the example in Section 4 is invalid. Yet, the main message of the paper is unchanged: triangular dynamic stochastic optimization problems with certain structure can be solved with the multidimensional version of the endogenous grid point method without any root-finding operations.

\section{References}

Iskhakov, F., 2015. Multidimensional endogenous dridpoint method: Solving triangular dynamic stochastic optimization problems without root-finding operations. Econom. Lett. 135, 72-76. 